\title{
Archéopages
}

Archéopages

Archéologie et société

Hors-série 3 | 2012

Nouveaux champs de la recherche archéologique

\section{L'archéologie préventive à la (re)découverte du peuple goth en Gaule du sud}

Jean-Luc Boudartchouk, Jérôme Hernandez, Didier Paya et Christian Scuiller

\section{(2) OpenEdition}

12 Journals

Édition électronique

URL : https://journals.openedition.org/archeopages/715

DOI : 10.4000/archeopages. 715

ISSN : 2269-9872

Éditeur

INRAP - Institut national de recherches archéologiques préventives

Édition imprimée

Date de publication : 1 janvier 2012

Pagination : 164-169

ISSN : 1622-8545

\section{Référence électronique}

Jean-Luc Boudartchouk, Jérôme Hernandez, Didier Paya et Christian Scuiller, «L'archéologie

préventive à la (re)découverte du peuple goth en Gaule du sud », Archéopages [En ligne], Hors-série 3 |

2012, mis en ligne le 01 janvier 2012, consulté le 24 février 2023. URL : http://

journals.openedition.org/archeopages/715; DOI : https://doi.org/10.4000/archeopages.715 

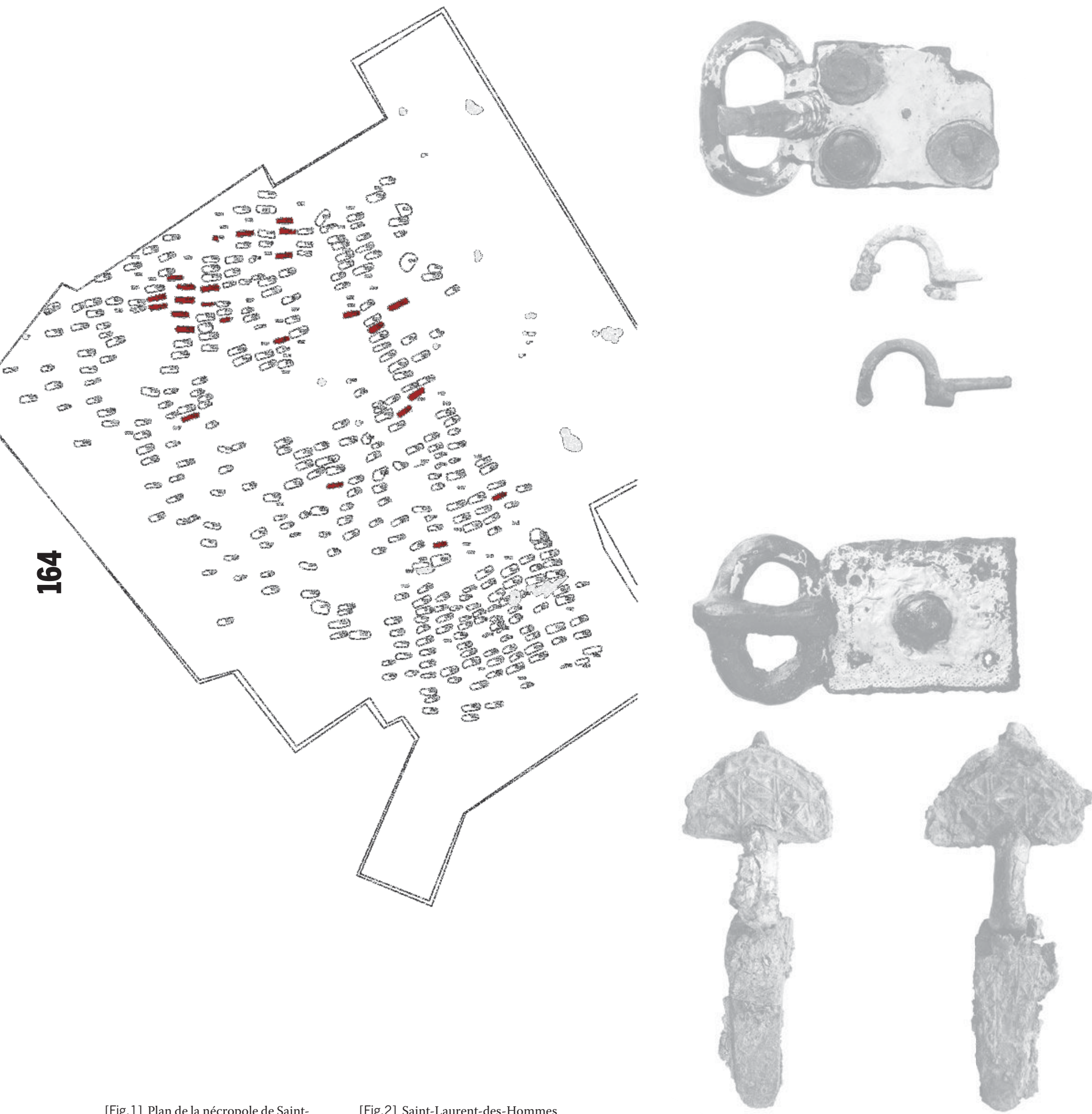

[Fig. 1] Plan de la nécropole de SaintLaurent-des-Hommes avec identification des tombes ayant livré du mobilier goth caractéristique (en rouge).
[Fig 2] Saint-Laurent-des-Hommes (Dordogne), mobilier de la sépulture féminine 1195 : plaque-boucle et fibules.

[Fig.3] Saint-Laurent-des-Hommes, mobilier de la sépulture féminin 1427 : plaque-boucle et fibules.

[Fig.4] Saint-Laurent-des-Hommes, mobilier de sépulture féminine 1519 : plaque-boucle.

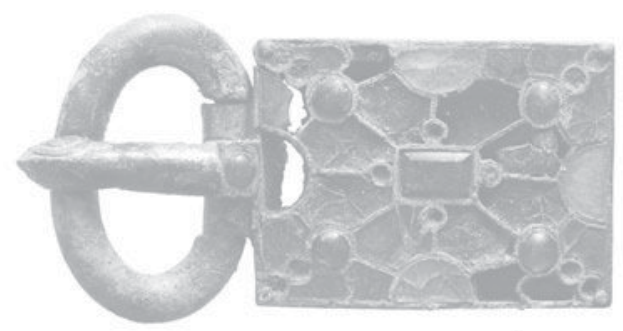


notamment au travers du droit et des institutions militaires, une sorte de « citoyenneté » au-delà des deux peuples en voie de rapprochement. Des processus que l'on avait vu à l'œuvre, initiés par Rome, quelques siècles auparavant....

BARRIÈRE-FLAVY C., 1892 : Étude sur les sépultures barbares du Midi et de l'Ouest de la France. Industrie wisigothique, Toulouse-Paris. Drioux ET Leroy, 1875 : Atlas universel et classique de géographie ancienne, romaine, du Moyen Age, moderne et contemporaine, Paris.

BARBERO A., 2009 : Barbares. Immigrés, réfugiés et déportés dans l'Empire romain, Tallandier, coll. Antiquité, Paris, $351 \mathrm{p}$.

BAILly P., 1984: «Une mention de « Patrice » dans une sépulture du $\mathrm{V}^{\mathrm{e}}$ siècle à Bourges ", in Actes du XLIII Congrès de la Fédération des sociétés savantes du Centre-Etudes creusoises V, Guéret, p. 39-43.

BOUDARTCHOUK J.-L., 2009: "Toulouse, de la ville wisigothique à la ville franque ( $\mathrm{v}^{\mathrm{e}}$-viII ${ }^{\mathrm{e}}$ siècle). Histoire et archéologie», in Toulouse, une métropole méridionale : vingt siècles de vie urbaine, Toulouse, éd. Méridiennes/Fédération historique de Midi-Pyrénées, vol. 1, p. 31-47.

Boudartchouk J.-L., Arramond J.-C., Grimbert L., 2006 : «Une sépulture de relégation découverte dans un fossé antique sur le site de l'Ilôt Castelbou (Toulouse, 1991) : un guerrier Vandale inhumé à la hâte? ", De l'âge du Fer au haut Moyen Age. Archéologie funéraire, princes et élites guerrières, t. XV des Mémoires de l'A.F.A.M., Condésur-Noireau, p. 122-133.

Brodeur J., Mortreau M., YvineC J.-H., 2001 : «Présence d'auxiliaires sur le site d'Angers. Mise en persbective au travers des fouilles d'Angers», in Militaires «barbares» dans l'armée romaine en Occident. Actualités archéologiques du haut Moyen Âge dans l'Ouest de la France, Pré-actes des XXII journées internationales d'archéologie mérovingienne, Angers, p. 9-12.

BUHOT DE KERSERS, 1892 : «Sépulture mérovingienne du cimetière des Capucins », in Mém. Soc. Ant. Centre, t. XVIII, 1891, Bourges, p. 51-63.

Demougeot E., 1979, La Formation de l'Europe et les invasions barbares, vol 2 : De l'avènement de Dioclétien au début du vi siècle, Aubier, Paris, $520 \mathrm{p}$.

De FILIPPO R., 1996 : « Le grand bâtiment du site de Larrey : la question palatiale», in Aquitania, XIV, p. 23-29.

Delaplace Ch., 2010 : «Le royaume wisigothique de Toulouse », in Wisigoths et Francs autour de la bataille de Vouillé (507). Recherches sur le haut Moyen Age dans le Centre-Ouest de la France, Actes des XXII Journées internationales d'archéologie mérovingienne (Vouillé et Poitiers, 28-30 septembre 2007), éd. AFAM, SaintGermain-en Laye, p. 15-18.

GofFART W., 1980 : Barbarians and Romans A. D. 418-584: the Techniques of Accommodation, Princeton University Press, Princeton, $279 \mathrm{p}$

KAZANSKI M., 1991 : Les Goths ( $I^{e r}-V I I^{e}$ après J.-C.), Paris, éd. Erance.

KAZANSKI M., LAPART J., 1995 : «Quelques documents du v ${ }^{\mathrm{e}}$ siècle ap. J.-C. attribuables aux Wisigoths découverts en Aquitaine», in Aquitania, XIII, p. 193-202.

LABrousse M., 1968 : Toulouse antique des origines à l'établissement des Wisigoths, Paris, École Française de Rome, 212.

LOYEN A., 1934: «Les Débuts du royaume wisigoth de Toulous », Revue des études latines, p. 406-415.

Mathisen R. W., 1999: Sivan H.S., «Forging a New Identity : the Kingdom of Toulouse and the Frontiers of Visigothic Aquitania (418-507)", in The Visigoths. Studies in Culture and Society, FERrEIro A. (ÉD.), Leiden-Boston-Köln, p. 2-62.

PÉRIN P., KAZANSKI M., 2011 : «Identity and Ethnicity during the Era of Migrations and Barbarian Kingdoms in the Light of Archaeology in Gaul», in Romans, Barbarians and the Transformation of the Roman World, Mathisen R.W. et Shanzer D. (éd.), Ashgate, p. 299-329.

PRÉVÔT F., 1997 : Recueil des inscriptions chrétiennes de la Gaule antérieures à la Renaissance carolingienne, t. 8 (Première Aquitaine), Paris, $168 \mathrm{p}$

Rouche M., 1979, L'Aquitaine des Wisigoths aux Arabes 418-781: naissance d'une nation, École des Hautes Études en Sciences Sociales, J. Touzot (éd.), Paris, 778 p.

Sivan H., 1984 : On Foederati, Hospitalitas and the settlement of the Goths in A.D. 418, in American Journal of Philology, vol. 108/4, p. 759-772.

Teillet S., 1984 : Des Goths à la nation gothique. Les origines de l'idée de nation en Occident du $V^{e}$ au VII siècle, coll. Études anciennes, Les Belles Lettres, Paris, 687 p.

WARD-PERKINS BR., The fall of Rome and the end of civilization, Oxford, 2005

Wolfram H., 1991, Histoire des Goths, coll. L'évolution de l'humanité, Albin Michel, Paris, 574 p.
L'archéologie préventive à la (re)découverte du peuple goth en Gaule du sud

\author{
Jean-Luc Boudartchouk \\ Jérôme Hernandez \\ Didier Paya \\ Christian Scuiller \\ Inrap
}

L es textes contemporains, si les mots latins qui nous sont parvenus ont un sens, sont sans ambiguïté sur un point : le peuple goth, qui prend possession de la Gaule du sud à partir de 413 et jusqu'en 508, constitue bien une entité sociologique et culturelle distincte au sein de l'ensemble de la population et son poids démographique, s'il est difficile à évaluer précisément, n'est pas négligeable. Or pendant longtemps les chercheurs ont eu du mal à caractériser de manière catégorique les vestiges archéologiques qui pouvaient leur être attribués, ce qui a conduit Edward James à conclure, en 1977, que sans les sources historiques la présence wisigothique en Gaule serait passée totalement inaperçue (James 1977). Jusqu'à une date récente, la majorité des chercheurs est restée fidèle à ce postulat, invoquant diverses raisons pour expliciter ce qui constitue tout de même un extraordinaire paradoxe : plus de cent mille, voire plusieurs centaines de milliers d'individus non romains, présents durant quatre générations en Gaule méridionale et centrale, n'auraient laissé aucune trace. On a évoqué (en minorant très fortement les chiffres donnés par les sources antiques) leur faible nombre au regard de la population romaine des provinces d'Aquitaine et de Narbonnaise. On a mis en avant l'état d'acculturation avancé des Goths à leur arrivée en Gaule : leur parcours chaotique dans le barbaricum puis à travers l'Empire romain d'est en ouest, les aurait peu à peu transformé en une armée errante (Wolfram, 1990). Cette mutation se serait accomplie en une seule génération, et serait conjointe à une transformation radicale de la structure même de la population (Kazanski 2010), tant au niveau de sa composition biologique que de sa stratification sociale (abandon d'éléments caractéristiques des sociétés germaniques non romanisées). Cette acculturation se serait accentuée pendant leur installation en Gaule : leur statut de militaires au service de l'Empire les aurait exclus des activités économiques et les aurait «obligés » à user d'objets de la vie quotidienne produits par les artisans locaux et à habiter dans les bâtiments construits selon des savoir-faire locaux. De manière générale, la plupart des historiens, hormis quelques-uns (Rouche, 1979), ont prêté aux Goths la volonté (au moins apparente) de se fondre dans la romanité.

Les archéologues ont eu tendance à partir de ces postulats pour interpréter les rares vestiges attribuables aux Goths. Cette présumée perméabilité aux influences romaines transparaitrait tout particulièrement au travers des pratiques funéraires, quasi absentes des textes mais révélées de manière spectaculaire par l'archéologie. Les Goths semblent 


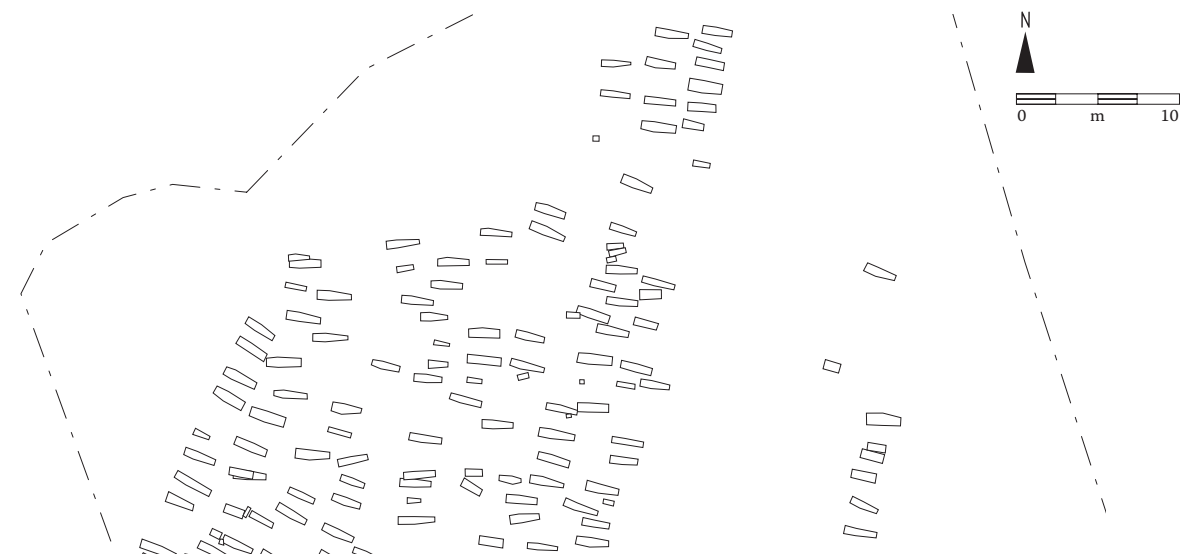

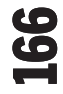

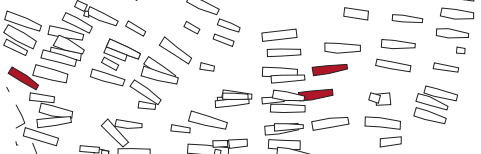

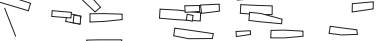

एक $\square \cong$

, $\square$

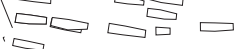

50

$\checkmark \square \square \square$

$\square \square$

$\square_{\square} \square$

i $\square$

$<\rightarrow \square$

$\downarrow$

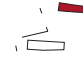

10

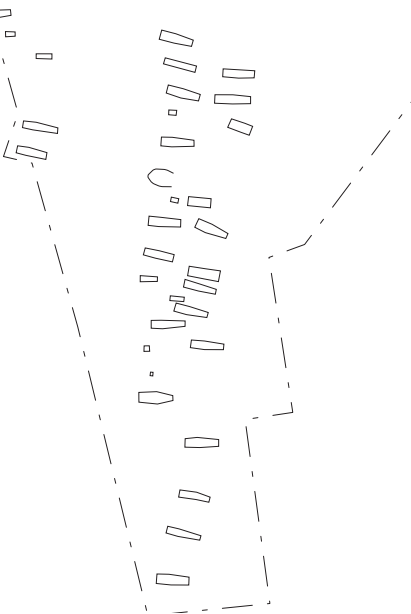

[Fig.5] Plan de la nécropole du Mouraut avec identification des tombes ayant livré du mobilier wisigothique (en rouge).
[Fig.6] Mobilier wisigothique du

Mouraut : fibules et plaques-boucles.

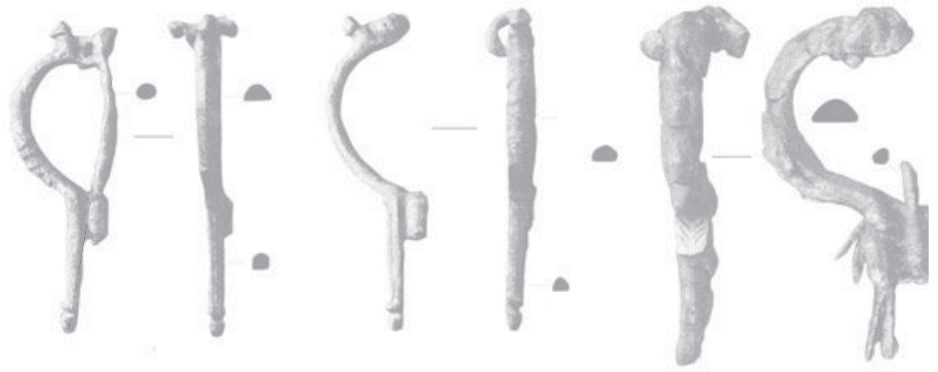


adopter les mêmes pratiques funéraires que les Gallo-Romains. La pratique de l'inhumation avec mobilier de parure, voire avec mobilier d'accompagnement, attestée chez les Goths pour le premier tiers du $\mathrm{v}^{\mathrm{e}}$ siècle $\mathbf{1}$, aurait été abandonnée rapidement au profit de la coutume romaine de l'inhumation sans mobilier. Cependant, pour la fin $\mathrm{du} \mathrm{v}^{\mathrm{e}}$ siècle, les archéologues ne peuvent que constater le retour à l'utilisation d'éléments d'identification caractéristiques du costume goth «évolué» (fibules, éléments de ceinture...).

Mais l'identité culturelle de ces porteurs/ porteuses posait problème : Goths de troisième ou quatrième générations et/ou Romain(e)s acculturé(es) ? Ce débat, parfois vif, concernait un faible nombre d'inhumations (découvertes isolées ou anciennes) ne fournissant qu'une information limitée. Or, récemment, plusieurs fouilles préventives ont permis de renouveler très sensiblement ce corpus et d'apporter de précieux éléments sur la diffusion de la culture gothe au sein de l'entité politique (regnum) circonscrite par la Loire (voire au-delà vers le nord ?) et le Rhône, et dont la capitale est Toulouse. Le vaste ensemble funéraire de SaintLaurent-des-Hommes ${ }^{2}$, en Dordogne, présente, à première vue les caractéristiques habituelles d'une nécropole du sud de la Gaule du début du $\mathrm{V}^{\mathrm{e}}$ siècle au début du $\mathrm{VI}^{\mathrm{e}}$. Les 364 tombes relevées (fosses oblongues de $0,15 \mathrm{~m}$ à $0,90 \mathrm{~m}$ de profondeur) sont orientées est-ouest et organisées en grands alignements constituant autant de rangées est-ouest que nord-sud [Fig.1]. Les défunts devaient y être placés dans des cercueils de bois cloués (traces de bois et/ou des clous dans plusieurs fosses). Cette nécropole se développe à l'extrémité d'une butte, sur une surface d'environ $4.000 \mathrm{~m}^{2}$, à proximité de structures en creux datées de la période mérovingienne. Mais le mobilier découvert dans 113 inhumations permet de restituer très vraisemblablement l'installation d'une communauté « exogène » (scil. d'origine gothe), intrinsèquement homogène : aucune arme, de rares objets utilitaires (principalement fermoirs d'aumônière et couteaux), nombreux éléments de parure [Fig.2,3,4] (essentiellement plaquesboucles, boucles, fibules). Dans certaines tombes, la concentration de perles minuscules, de couleur verte, corail, or ou noire, pourrait indiquer la présence d'éléments confectionnés tels que des coiffes ou des colliers dont les parallèles connus de nous, bien qu'assez lointains, sont situés en Espagne wisigothique et en Europe orientale. Le mobilier de parure met en relief un dimorphisme sexuel dans le costume particulièrement frappant avec, d'un côté, les grandes parures typiquement wisigothiques (fibules et plaque-boucle) dans des sépultures féminines, et, de l'autre, des tombes masculines caractérisées par des éléments de ceinturon pour lesquels, au-delà de l'aire d'influence des Goths de Gaule, on connait des parallèles au sein d'autres groupes militarisés, romains ou non-romains, certains datant du premier tiers du v $\mathrm{V}^{\mathrm{e}}$ siècle (Böhme, 1974). Un faisceau d'indices convergents (chronotypologie et associations de mobiliers caractéristiques) permet, dans ce cas précis et sans doute pour la première fois en Gaule du sud, d'émettre l'hypothèse d'un groupe humain homogène composé de Goths, inhumés sur le même site durant un siècle, depuis les années 410 (au lendemain de leur installation) jusqu'au début $\mathrm{du} \mathrm{VI}^{\mathrm{e}}$ siècle (au moment de la destruction de leur regnum par les Francs en 507-508). En effet, un changement radical s'opère alors dans la composition des ensembles mobiliers au cours de la première moitié du $\mathrm{VI}^{\mathrm{e}}$ siècle. Les parures wisigothiques disparaissent au profit de garnitures de ceinturon relevant désormais exclusivement du costume franc. Sans doute touche-t-on du doigt ici l'«archéologie du changement culturel».

Le cimetière du Mouraut ${ }^{\mathbf{3}}$, au sud de Toulouse, parait présenter un modèle d'implantation/ fonctionnement et peut-être, un mode de recrutement, différent. Là aussi, les 316 tombes s'organisent en rangées successives, sur une terrasse fluviatile émergente dans une zone marécageuse [Fig.5]. Les inhumations habillées représentent 4.0\% de l'effectif global. La zone explorée correspond à la partie orientale d'un vaste ensemble funéraire occupé du $\mathrm{V}^{\mathrm{e}}$ jusqu'au VIII ${ }^{\mathrm{e}}$ ou $\mathrm{IX}^{\mathrm{e}}$ siècle. La présence de sépultures habillées varie au long des quatre siècles d'occupation et n'est qu'un paramètre parmi d'autres, permettant cependant de révéler, au cours de la seconde moitié du v ${ }^{\mathrm{e}} \mathrm{s}$., l'arrivée d'un ou de plusieurs groupes humains « exogènes» (scil. nonromains). Plusieurs tombes appartenant au même horizon chronologique présentent un mobilier « oriental» (boucles d'oreilles de type nomade), ainsi que des déformations crâniennes. La répartition topographique des tombes pourrait indiquer que les ressortissants de ces groupes se mêlent à une population locale, avec laquelle ils ont en commun une partie des pratiques funéraires. Les inhumations habillées, ainsi que les sujets présentant une déformation crânienne, voisinent, sans que l'on puisse saisir de logique spécifique, les «autres» tombes dépourvues de mobilier. Enfin, l'ensemble de la population, de manière indifférenciée, repose dans des tombes de mêmes types : coffres de bois cloués ou non et, plus rarement, coffre de tegulae. Deux coffres en tuiles ont été mis au jour : l'un recelait un porteur d'arme mérovingien, l'autre une sépulture sans mobilier mais qui présente une déformation crânienne dont la pratique est généralement associée à des peuples « orientaux» (dont les Goths). En effet, ces défunts portent des objets caractéristiques de la culture matérielle des Goths du v viècle et/ou d'autres cultures « orientales» voisines [Fig.6].

Quelques inhumations, que l'on peut situer au début $\mathrm{du} \mathrm{VI}^{\mathrm{e}}$ siècle, recèlent des associations de mobiliers spécifiques attestant du port du « costume franc », faciès mis en évidence auparavant sur le site de La Gravette (Ictium) à l'Isle-Jourdain. Le site du «Mouraut» peut sans doute être appréhendé comme l'illustration archéologique d'un fait historique marquant, la conquête franque, puisque c'est peu après 500 et en tout cas au cours de la première moitié du vi ${ }^{\mathrm{e}} \mathrm{s}$., que les grandes parures wisigothiques y disparaissent.

Les apports, acquis et à venir, de l'étude des cimetières de Saint-Laurent-des-Hommes et 
du Mouraut du peuplement de l'Aquitaine gothe à la veille de la bataille de Vouillé (507), sont d'ores et déjà majeurs. Ils éclairent de façon nouvelle certains aspects des modalités d'installation et d'intégration des nouveaux venus et obligent à intégrer la présence avérée, au cœur du regnum, de groupes humains culturellement homogènes (et non plus seulement d'individus ou de «familles»), de véritables communautés non romaines implantées en milieu rural : les Goths.

вӧнмЕ H.W., 1974 : Germanishe Grabfunde des 4. Bis 5. Jahrunderts zwischen unteren Elbe und Loire, Beck, Munich, 2 vol.

BOUDARTCHOUK J.-L., 1998 : «La nécropole franque d'Ictium à L'IsleJourdain (Gers, Midi-Pyrénées) », in Die Franken - Les Francs, Acta Praehistorica et Archaeologica, 3o, Berlin, p. 126-136.

JAMES E., 1977 : The merovingian archaeology of south-west Gaul, British Archaeological reports, Supplementary series 25, Oxford, 2 vol.

KAZANSKI M., 2010 : «Les Wisigoths, du Danube à la Gaule (375-412)» in Bourgeors L. (DIR.) Wisigoths et Francs autour de la bataille de Vouillé (507): Recherches récentes sur le haut Moyen Age dans le Centre-Ouest de la France, Actes des XXVIII journées internationales d'archéologie mérovingienne, Saint-Germain-enLaye, p. 9-14.

Rouche M., 1979: L'Aquitaine des Wisigoths aux Arabes 418-781: naissance d'une nation, École des Hautes Études en Sciences Sociales, J. Touzot (éd.), Paris, 778 p.

Wolfram H., 1991 : Histoire des Goths, Albin Michel, coll. Lévolution de l'humanité, Paris, 574 p.

\section{Mal enterrés et mal rangés : le cas des squelettes humains sans sépulture en France gallo-romaine et médiévale}

Isabelle Rodet-Belarbi

Inrap, UMR 7264 «Cultures-Environnements, Préhistoire, Antiquité, Moyen Âge »

Isabelle Séguy

INED, UMR 7264 «Cultures-Environnements, Préhistoire,

Antiquité, Moyen Âge »

$\mathrm{L}$

es nombreuses fouilles en contextes archéologiques variés, couvrant de larges espaces et de longues périodes chronologiques, ont permis la découverte de vestiges très différents les uns des autres, dont certains témoignent de situations peu communes. L'une d'elles illustre un phénomène connu par les textes médiévaux ${ }^{\mathbf{1}}$ : celui des enfants mort-nés ou dont on voulait se débarrasser dès la naissance et qui ne recevaient aucune sépulture.

Ainsi, lorsque Cécile Treffort se demande à quoi correspondent les « corps abandonnés sans soin particulier à la merci des animaux sauvages ou

1 P.-A. Sigal mentionne le cas d'une habitante d'Aix-en-Provence ayant accouché de jumeaux mort-nés dont les corps furent jetés sur un tas de fumier (Sigal, 1987, p. 31) Des procès médiévaux et modernes relatent des cas d'infanticides ; les corps des nouveaunés ayant été jetés dans des latrines, des puits, des auges à cochons... 2 Le terme «périnatal » regroupe les enfants morts juste avant, pendant ou juste après leur naissance. à notre mantalité dur. Bien que cette «idée répugne par des témoignages médiévaux (Treffort, 1997, p. 101). En effet, « les pénitentiels du Haut Moyen Âge interdisent formellement de consommer des animaux, y compris des bêtes délevage, ayant mangé de la chair humaine. On peut se demander, avec P. Bonnassie, à qui appartiennent ces corps. S'agit-il entre autres, se demande-t-il, «d'enfants excédentaires, délibérément "perdus", de nouveau-nés exposés » ou, pourrions-nous ajouter, d'enfants morts en bas âge et non ensevelis ? (Treffort, 1997, p. 102).

Des découvertes archéologiques montrent que, si certains nouveau-nés sont enterrés dans les nécropoles ou les cimetières, d'autres le sont au sein des habitats, dans des sépultures inhabituelles. Il en est d'autres encore qui, manifestement, n'ont eu droit à aucun égard et ont été jetés parmi les rejets domestiques. Nous en avons des exemples qui, par leur similitude, nous ont permis de proposer la notion de «non-sépulture» (Rodet-Belarbi, Séguy, à paraître ; Séguy, Rodet-Belarbi, à paraître) et de répondre par l'affirmative au questionnement des historiens. En effet, des squelettes de périnataux ${ }^{2}$ ont été jetés dans des dépotoirs, des décharges, des puits en cours de comblement... et mis au jour au beau milieu des lots de faune. Comme il s'agit d'individus complets, et non d'os erratiques résultant de l'écrêtage de nécropoles et de cimetières antérieurs, nous y avons vu un geste volontaire. Il était nécessaire de disposer de la double détermination homme/ animal pour que notre analyse ne soit pas biaisée et repose sur des informations exhaustives. Ce faisant, nous nous sommes aperçu que ce phénomène n'était pas exclusivement réservé aux périnataux, mais que d'autres groupes d'âges pouvaient être concernés. Un tel phénomène est illustré par diverses découvertes en France.

Les individus périnataux. Deux sites éloignés géographiquement, mais proches chronologiquement, Jublains (Mayenne ; Bocquet et al., 2004) et Narbonne (Aude; Sabrié, 2011), présentent des caractéristiques communes [Fig.1]. Sur ces deux sites, occupés dès le début du I ${ }^{\text {er }}$ siècle de notre ère, des puits à eau ont été creusés au sein d'un quartier artisanal, dans le premier cas, et au centre d'une domus, dans le second.

Lors de son abandon, daté de la fin du III ${ }^{\mathrm{e}}$-début du IV ${ }^{\mathrm{e}}$ siècle, le puits PT 3082 de Jublains a été essentiellement comblé de déchets artisanaux, de restes osseux correspondant à des reliefs de repas, de squelettes animaux et humains. Des cadavres de chiens de tous âges, de deux chèvres, d'un équidé adulte, ainsi que les ossements d'un périnatal âgé de 9-10 mois lunaires ont été identifiés (Forest, Fabre [coll.], 1999, p. 8). Malgré un bon état de conservation et le tamisage des sédiments de la moitié inférieure du remplissage, les os humains et animaux de petite taille n'ont pas été retrouvés.

Le puits de la maison au grand Triclinium de Narbonne, abandonné au début du III ${ }^{\mathrm{e}}$ siècle et comblé au cours du même siècle, a été rempli de déchets divers : céramiques, détritus alimentaires et os erratiques de diverses espèces consommées (porc, mouton/chèvre, cerf, lapin de garenne, oiseaux et poissons). Comme à Jublains, divers squelettes y ont été découvert : chiens immatures et adultes, jeune équidé, singe magot (Macaca sylvana) et aussi enfants morts en période périnatale (Forest, Fabre, 2011). À nouveau, malgré l'absence de leurs os les plus petits, tout porte à croire que ces squelettes humains étaient complets lors de leur rejet dans le puits. Les os rochers ont permis d'estimer à 27 le nombre minimum d'individus. Ce chiffre peut paraître très important au regard de la séquence d'utilisation de ce puits comme dépotoir, qui n'a duré que quelques

dizaines d'années dans un quartier en cours 